\title{
A kinetic modeling for the ultrasound-assisted and oxalic acid-catalyzed hydrolysis of 3-glycidoxypropyltrimethoxysilane
}

\author{
Dimas R. Vollet $^{1}$ (1) Luiz A. Barreiro $^{1} \cdot$ Carlos E. T. Paccola $^{1} \cdot$ Carlos M. Awano $^{1}$ \\ Fabio S. de Vicente ${ }^{1} \cdot$ Makoto Yoshida $^{1}$. Dario A. Donatti ${ }^{1}$
}

Received: 20 April 2016/ Accepted: 14 July 2016/Published online: 20 July 2016

(c) Springer Science+Business Media New York 2016

\begin{abstract}
Instantaneous hydrolysis rates of the ultrasoundassisted and oxalic acid-catalyzed hydrolysis of 3-glycidoxypropyltrimethoxysilane (GPTMS) have been obtained at several temperatures by using a dynamic ultrasoundadapted calorimetric method. Hydrolysis starts by ultrasound action because of the initial immiscibility gap between GPTMS and water. The hydrolysis process is a complex result of dissolution between GPTMS and $\mathrm{H}_{2} \mathrm{O}$, which increases the hydrolysis rate, and reaction within the phases, which diminishes the hydrolysis rate as the reactants are consumed. The experimental hydrolysis rates were very well fitted by a modified version of an earlier kinetic model based on a dissolution and reaction mechanism. The rate constants for the ultrasound and methanol producing dissolution and the rate constants for the GPTMS hydrolysis were obtained by fitting the modeling to the overall heterogeneous/homogeneous hydrolysis pathway at each temperature studied. The hydrolysis rate constants were found in good agreement with those obtained previously on basis of a non-modeling method applied exclusively to the final homogeneous step of the reaction. Ultrasound producing mixture was found much more effective than methanol producing dissolution during the heterogeneous step of the GPTMS hydrolysis.
\end{abstract}

Dimas R. Vollet

vollet@rc.unesp.br

1 Departamento de Física, IGCE, Unesp - Univ Estadual Paulista, Cx.P. 178, Rio Claro, SP 13500-970, Brazil
Graphical Abstract

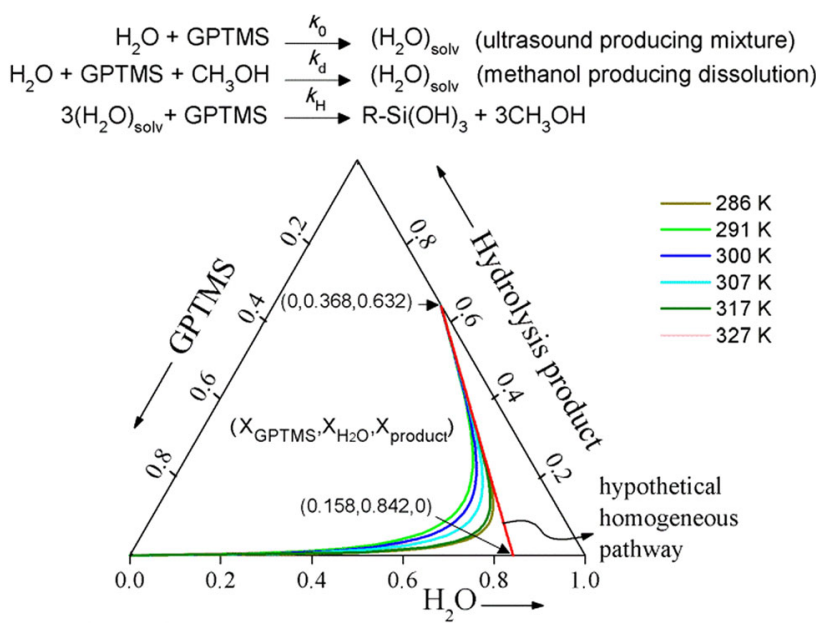

Keywords 3-Glycidoxypropyltrimethoxysilane .

Ultrasound and hydrolysis · Oxalic acid · Kinetic modeling

\section{Introduction}

3-Glycidoxypropyltrimethoxysilane (GPTMS) is an interesting trialkoxysilane precursor for the preparation of hybrid organic-inorganic materials because it possesses functionality of both silicon alkoxide and an epoxy ring [1-4]. An important feature of the organic-functionalized alkoxides polymerization is competition between the ongoing processes of hydrolysis, condensation and phase separation, which determines the final properties of the hybrid [5, 6]. GPTMS has been applied in a variety of areas like proton-conducting membranes, corrosion and scratchresistant coatings and optical applications, mainly due to its cross-linking capacity through the epoxy group [2]. 
The sol-gel polymerization of GPTMS involving hydrolysis and polycondensation of monomers often leads to the formation of fully condensed structures of formula $\left(\mathrm{RSiO}_{3 / 2}\right)_{n}$ or partially condensed structures of formula $\left[\mathrm{RSiO}_{3 / 2-x}(\mathrm{OH})_{2 x}\right]_{m}$ (where $\mathrm{R}$ is 3-glycidoxypropyl radical) [7], leading to different structures for the resulting networks, such as 3D networks of trifunctional monomers, "ladder" structures, cake-like fragments, and a combination of linear, "ladder" and cake-like fragments [1, 7-9], depending on the polymerization conditions. The organicinorganic condensation and the network formation depend on the $\mathrm{pH}$ conditions [10], so hybrid organic-inorganic materials synthesized from GPTMS in strong basic conditions can even present crystalline forms ruled mainly by the epoxy-opening reaction $[10,11]$, or only a poor degree of self-organization and crystallization by lowering the $\mathrm{pH}$ $[1,9,10]$.

Although the organic-inorganic condensation and the network formation of GPTMS have been studied at some extension, as far we know, there are few works dealing with the hydrolysis of GPTMS. Since GPTMS and water are immiscible, generally methanol is used as a co-solvent in the conventional GPTMS hydrolysis. However, methanol is also a by-product of the condensation reactions in the sol-gel synthesis and it could lower the control degree of the final product [1]. Ultrasound is an alternative method to promote hydrolysis of alkoxysilanes without using alcoholic co-solvents [12, 13]. Ultrasound not only accelerates the hydrolysis reaction but can modify the steps required for conventional reactions, without the necessity of using alcoholic solvents that could influence condensation reactions later, so diminishing the control degree of the final product. For instance, sonogels prepared from solventless hydrolysis of silicon alkoxides by ultrasound stimulation generally exhibit structure formed by a finer porosity and particles with more homogeneous size distribution in comparison with those structural properties of conventional gels prepared with alcoholic solvents [12]. In a previous work [14], we have studied the ultrasound-assisted and oxalic acid-catalyzed hydrolysis of GPTMS by measuring the instantaneous hydrolysis rates by means of an ultrasound-adapted dynamic calorimetric method. The data were interpreted in terms of a two-steps process: An initial heterogeneous step in which the reactants GPTMS and water remain not completely mixed, and a final homogeneous step in which the hydrolysis rate equation was found to be well described by a 3/4-order rate constant with respect to GPTMS and first order with respect to water. Oxalic acid, besides its acid catalytic effect on the GPTMS hydrolysis, may also modify the organic-inorganic condensation and the network formation by means of several possible mechanisms of epoxy ring opening $[15,16]$, often occurring after hydrolysis, increasing the possibilities of formation of different types of cross-linked organic structures.

In this work, we applied a modified version an earlier dissolution and reaction kinetic modeling [17, 18] to describe the set of the experimental ultrasound-assisted and oxalic acid-catalyzed GPTMS hydrolysis rates, as measured at several temperatures. The hydrolysis rate constants and the rate constants for the ultrasound and methanol producing dissolution were obtained by fitting the modeling to the overall heterogeneous/homogeneous hydrolysis pathway, and the results were discussed.

\section{Experimental}

The hydrolysis of 3-glycidoxypropyltrimethoxysilane (GPTMS) under ultrasound stimulation was carried out in about $36 \mathrm{~mL}$ reactant mixtures prepared with $25 \mathrm{~mL}$ of GPTMS (Aldrich $98 \%$ ), $6 \mathrm{~mL}$ of distilled and deionized water, and $5 \mathrm{~mL}$ of $0.3 \mathrm{M}$ oxalic acid, as a catalyst. The nominal initial concentrations of the reagents in the mixtures were $A_{0}=3.15 \mathrm{M}$ for GPTMS, $B_{0}=16.8 \mathrm{M}$ for water, and $M_{0}=0.042 \mathrm{M}$ for the catalyst, which gives a theoretical $\mathrm{pH} \cong 1.57$, as evaluated from $\left[\mathrm{H}^{+}\right]=(1 / 2)\left[-K_{\mathrm{a} 1}+\right.$ $\left.\left(K_{\mathrm{a} 1}^{2}+4 K_{\mathrm{a} 1} M_{0}\right)^{1 / 2}\right]$, where $\mathrm{p} K_{\mathrm{a} 1}=1.27$ is the first ionization constant for the oxalic acid at $298 \mathrm{~K}$, being $K_{\mathrm{a} 1} \gg K_{\mathrm{a} 2}$ [19]. The hydrolysis rates as a function of the sonication time were obtained by measuring the thermal peak produced by the rapid GPTMS hydrolysis (occurring typically in about $1 \mathrm{~min}$ ) in an ultrasound-adapted dynamic heat-flow calorimeter. For the sonohydrolysis, a constant power of ultrasound releasing about $0.7 \mathrm{~W} / \mathrm{cm}^{3}$ to the reactant mixture was applied through of a 10-mm diameter transducer tip by using a $100 \mathrm{~W}$ US-power Unique equipment operating at $19 \mathrm{kHz}$. It should be pointed that addition of the catalyst without sonication (or with conventional mechanical stirring) could not start the reaction efficiently because of the immiscibility gap of the system. The characteristics of the ultrasound-adapted devise for the dynamic heat-flow calorimetry, the theoretical basis for the interpretation of the hydrolysis experimental data, and the experimental ultrasound-assisted hydrolysis procedure were described in detail elsewhere [14]. Instantaneous hydrolysis rates of GPTMS in absolute units of $\mathrm{M} \mathrm{s}^{-1}$ have been obtained experimentally at several temperatures. Under the experimental conditions employed, the measured thermal signal was assigned to the GPTMS hydrolysis according to the overall stoichiometric reaction

$\mathrm{R}-\mathrm{Si}\left(\mathrm{OCH}_{3}\right)_{3}+3 \mathrm{H}_{2} \mathrm{O} \rightarrow \mathrm{R}-\mathrm{Si}(\mathrm{OH})_{3}+3 \mathrm{CH}_{3} \mathrm{OH}$,

where $\mathrm{R}-\mathrm{Si}(\mathrm{OH})_{3}$ (being $\mathrm{R}$ the 3-glycidoxypropyl group) represents a fully hydrolyzed GPTMS molecule, instead of 
a partially hydrolyzed $\mathrm{R}-\mathrm{Si}\left(\mathrm{OCH}_{3}\right)_{3-x}(\mathrm{OH})_{x}$ one, since the colorimetric method employed could not distinguish a fully hydrolyzed GPTMS molecule from a set of three monohydrolyzed ones. So, the kinetic parameters probed in present work associated with the hydrolysis process should be interpreted as average values mediated on a fully hydrolyzed GPTMS molecule.

\section{Results and discussion}

\subsection{Experimental sono-hydrolysis rates}

Figure 1 shows the experimental hydrolysis rates $\mathrm{d} C /$ $\mathrm{d} t$ [where $C$ represents the concentration of the hydrolyzed $\mathrm{R}-\mathrm{Si}(\mathrm{OH})_{3}$ quantity according to Eq. (1)] as a function of the reaction time $t$ measured at several temperatures.

The experimental hydrolysis rates in Fig. 1 have been interpreted as the following: (1) There is a heterogeneous step during which GPTMS and water remain not completely mixed, in which $\mathrm{d} C / \mathrm{d} t$ increases with the ultrasound action and methanol yielded producing dissolution and

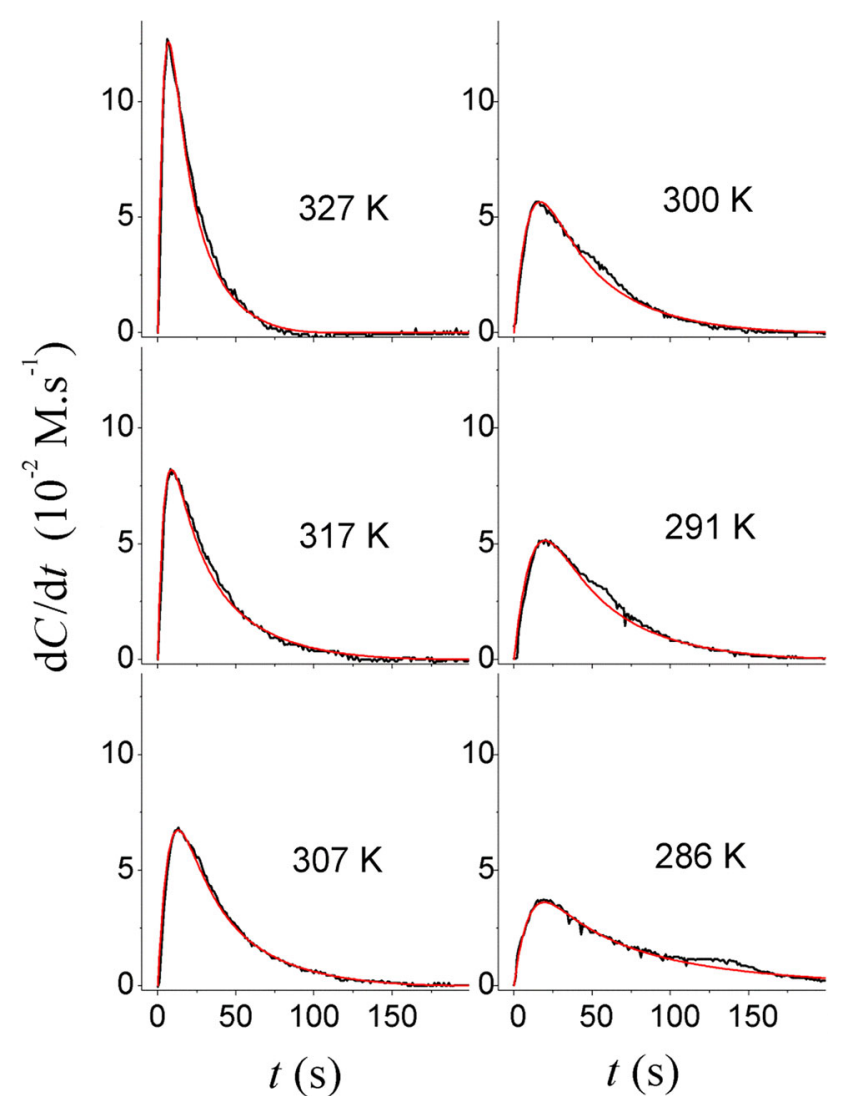

Fig. 1 Ultrasound-assisted and oxalic acid-catalyzed GPTMS hydrolysis rates as a function the reaction time as obtained at several temperatures. Full (red) lines are fittings of the present kinetic modeling (Color figure online) deaccelerates with the reaction within the phases and (2) a final homogeneous step when the system crosses the immiscibility gap, so $\mathrm{d} C / \mathrm{d} t$ decreases naturally as the reactants are consumed in a simple homogeneous pathway. It has been established [14] that the hydrolysis rate equation in the final homogeneous step of the reaction could be well described by a 3/4-order rate constant with respect to GPTMS and first order with respect to water. In order to minimize the parameters to be fitted, such a 3/4-order hydrolysis rate constant with respect to GPTMS and first order with respect to water was assumed in the hydrolysis rate equation of the present kinetic modeling.

\subsection{The kinetic modeling}

The characteristics of the present experimental hydrolysis rates giving rise to a heterogeneous and a homogeneous step of the reaction are typical of immiscible alkoxidewater systems. Such characteristics have been described earlier by a simplified dissolution and reaction kinetic model [17, 18], which has been applied to the ultrasoundassisted hydrolysis of the tetraethoxysilane (TEOS) [17] and tetramethoxysilane (TMOS) [18], carried out under acid conditions and excess of water.

A modified version of the mentioned kinetic modeling was considered to describe the present GPTMS hydrolysis rates as a time function. Since GPTMS and $\mathrm{H}_{2} \mathrm{O}$ are immiscible, the hydrolysis reaction of Eq. (1) could not be started, but very slowly at the interface between the phases GPTMS and $\mathrm{H}_{2} \mathrm{O}$. Ultrasound enhances efficiently the virtual dissolution between GPTMS and $\mathrm{H}_{2} \mathrm{O}$ giving start rapidly to the hydrolysis reaction. Methanol yielded in the firstly hydrolysis enhances de dissolution between GPTMS and $\mathrm{H}_{2} \mathrm{O}$, increasing even more the hydrolysis rate within the phases GPTMS and $\mathrm{H}_{2} \mathrm{O}$. The hydrolysis rate is then a complex result of dissolution between GPTMS and $\mathrm{H}_{2} \mathrm{O}$ (which increases the hydrolysis rate) and reaction within the phases (which diminishes the hydrolysis rate as the reactants are consumed).

To simplify the problem, and because the initial volume of the GPTMS is about $70 \%$ of the total volume of the reactant mixture, we assume a mechanism of dissolution of $\mathrm{H}_{2} \mathrm{O}$ (B) in GPTMS (A), followed by reaction between dissolved $\mathrm{H}_{2} \mathrm{O}$ (Bs) and GPTMS, according to the following steps

$\mathrm{B}+\mathrm{A} \stackrel{k_{0}}{\rightarrow} \mathrm{Bs}$

$\mathrm{B}+\mathrm{A}+\mathrm{Ds} \stackrel{k_{\mathrm{d}}}{\rightarrow} \mathrm{Bs}$

$\mathrm{A}+3 \mathrm{Bs} \stackrel{k_{\mathrm{H}}}{\rightarrow} \mathrm{C}+3 \mathrm{Ds}$

where B represents non-dissolved water, A represents GPTMS, Bs represents water effectively dissolved in 
GPTMS, Ds and $\mathrm{C}$ represent methanol and $\mathrm{R}-\mathrm{Si}(\mathrm{OH})_{3}$ produced by reaction in the GPTMS phase, $k_{0}$ is the rate constant associated with ultrasound producing dissolution of B in A [Eq. (2a)], $k_{\mathrm{d}}$ is the rate constant associated with methanol producing dissolution of B in A [Eq. (2b)] and $k_{\mathrm{H}}$ is the rate constant of hydrolysis between $\mathrm{A}$ and $\mathrm{Bs}$ yielding $\mathrm{C}$ and Ds [Eq. (2c)]. In this simplified model, B (non-dissolved or free water) would work as a water source feeding the hydrolysis reaction [Eq. (2c)] by means of the two parallel dissolution processes given by Eqs. (2a) and (2b). Assuming second order (on B and A) for the rate constant $k_{0}$, third order (on $\mathrm{B}, \mathrm{A}$ and Ds) for the rate constant $k_{\mathrm{d}}$, and 3/4 order on $A$ and first order on Bs for the hydrolysis rate constant $k_{\mathrm{H}}$ (as mentioned), we should have the following set of rate equations for $\mathrm{Bs}$ and $\mathrm{C}$ productions:

$\mathrm{d} B_{\mathrm{s}} / \mathrm{d} t=\left[k_{0}+k_{\mathrm{d}} D_{\mathrm{s}}\right] A B-3 k_{\mathrm{H}} A^{3 / 4} B_{\mathrm{s}}$

and

$\mathrm{d} C / \mathrm{d} t=k_{\mathrm{H}} A^{3 / 4} B_{\mathrm{s}}$

where the notations in italic $A, B, B_{\mathrm{s}}, C$ and $D_{\mathrm{s}}$ states for the corresponding molar concentrations of the species A, B, Bs, $\mathrm{C}$ and Ds, as defined in Eqs. (2a)-(2c). The assumption of third order (on $\mathrm{B}, \mathrm{A}$ and $\mathrm{Ds}$ ) for the rate constant $k_{\mathrm{d}}$, although this model could not correspond to a real mechanistic event, seems natural from the dissolution Eq. (2b). As we will see, the influence of $k_{\mathrm{d}}$ on the overall process is small, so it would not be possible to study with some success the influence of assuming a partial order on methanol, for instance, for the rate constant $k_{\mathrm{d}}$. Under these conditions, the rate equation $\mathrm{d} B_{\mathrm{s}} / \mathrm{d} t$ would be governed by the production of Bs from free water $B$ [the first term on the right side of the rate Eq. (3a)] and consumption of Bs by three times the rate equation for hydrolysis reaction between $\mathrm{A}$ and $\mathrm{Bs}$ [the second term on the right side of Eq. (3a)]. The hydrolysis rate $\mathrm{d} C / \mathrm{d} t$ would be a result of such a complex mechanism, starting from the initial conditions at $t=0$ with $A=A_{0}$, $B=B_{0}, B_{\mathrm{s}}=0, C=0$ and $D_{\mathrm{s}}=0$, with the constraints $A=A_{0}-C, D_{\mathrm{s}}=3 C$ and $B=B_{0}-3 C-B_{\mathrm{s}}$ (imposed by the stoichiometry of the reaction). When $B \cong 0$ (or $B_{\mathrm{s}} \cong B_{0}-3 C$ ) at the immediacy of the immiscibility gap of the system, the rate Eq. (3a) becomes simply $\mathrm{d} B_{\mathrm{s}}$ l $\mathrm{d} t=3 k_{\mathrm{H}} A^{3 / 4} B_{\mathrm{s}}=3 \mathrm{~d} C / \mathrm{d} t$ and the hydrolysis rate $\mathrm{d} C / \mathrm{d} t$ [Eq. (3b)] follows approximately a homogeneous pathway, according to a simple rate equation cast by

$\mathrm{d} C / \mathrm{d} t=k_{\mathrm{H}}\left(A_{0}-C\right)^{3 / 4}\left(B_{0}-3 C\right)$.

The rate Eq. (4) has been applied earlier [14] to describe exclusively the final homogeneous step of the reaction.

The apparent best fitting of the experimental $\mathrm{d} C / \mathrm{d} t$ to the rate Eq. (3b) was carried out by solving the set of coupled rate Eqs. (3a) and (3b) for the rate constants $k_{0}, k_{\mathrm{d}}$ and $k_{\mathrm{H}}$ at each temperature studied, starting from the mentioned initial conditions and the constraining equations. Figure 1 shows the general good fittings of the present dissolution and reaction modeling to the experimental hydrolysis rates. Table 1 shows the set of the rate constants $k_{0}, k_{\mathrm{d}}$ and $k_{\mathrm{H}}$ fitted at each temperature. Figure 2 shows the fitted parameters $k_{0}, k_{\mathrm{d}}$ and $k_{\mathrm{H}}$ in an Arrhenius-type plot.

Figure 2 shows that the hydrolysis rate constants $k_{\mathrm{H}}$ obtained from the present dissolution and reaction modeling applied to the overall heterogeneous/homogeneous GPTMS hydrolysis are in good agreement with those obtained earlier [14], using a non-modeling method applied exclusively to the final homogeneous step of the reaction, where Eq. (4) applies. The activation energy $\Delta E=(20.1$ $\pm 3.9) \mathrm{kJ} / \mathrm{mol}$ for the overall heterogeneous/homogeneous GPTMS hydrolysis obtained from the present modeling (Fig. 2) was also found in good agreement with that earlier $(20.1 \pm 3.2) \mathrm{kJ} / \mathrm{mol}$ obtained from exclusively the final homogeneous step of the reaction [14].

Figure 2 also shows that, in general, the ultrasound producing dissolution $\left(k_{0}\right)$ and methanol producing dissolution $\left(k_{\mathrm{d}}\right)$ rate constants increase with increasing temperature. The increase in the rate constants with temperature is expected since the overall dissolution process should be enhanced with increasing temperature. The exception to this role seems to be represented by the values $k_{0}$ and $k_{\mathrm{d}}$ obtained at low temperatures (at the right side of Fig. 2), which, on the contrary, were found increasing with diminishing temperature. An explication for this observation with respect to $k_{0}$ could be associated with the ultrasound coupling with the reactant medium, which is expected to be a complex function of the medium viscosity and density. Then, the ultrasound coupling with the medium could well be enhancing with lowering temperature, so increasing the ultrasound producing dissolution efficiency at low temperature. This could influence indirectly the methanol producing dissolution mechanism, since the ultrasound action in presence of methanol could be accelerating additional water dissolution. Then, the behavior of $k_{0}$ and $k_{\mathrm{d}}$ with temperature shown in Fig. 2 could be a result from two concurrent effects: (1) the natural increase in solubility with increasing temperature, and (2) the worsening of the ultrasound coupling with the medium with increasing temperature. These two concurrent effects could well be responsible for the apparent minimum observed in both plots of $k_{0}$ and $k_{\mathrm{d}}$ in Fig. 2.

The variations of $k_{0}$ and $k_{\mathrm{d}}$ with temperature shown in Fig. 2 are very similar since both sets of parameters could be fitted by essentially parallel curves. Since both mentioned effects, increasing of solubility and worsening of the ultrasound coupling (with increasing temperature), could not be separated, just a minimum activation energy for the 
Table 1 Rate constants for the dissolution and reaction modeling

\begin{tabular}{llll}
\hline$T(\mathrm{~K})$ & $k_{0}\left(10^{-2} \mathrm{M}^{-1} \mathrm{~s}^{-1}\right)$ & $k_{\mathrm{d}}\left(10^{-2} \mathrm{M}^{-2} \mathrm{~s}^{-1}\right)$ & $k_{\mathrm{H}}\left(10^{-3} \mathrm{M}^{-3 / 4} \mathrm{~s}^{-1}\right)$ \\
\hline 286 & $3.2(3)$ & $1.1(3)$ & $1.3(1)$ \\
291 & $2.0(2)$ & $0.4(1)$ & $2.6(2)$ \\
300 & $2.6(3)$ & $0.4(1)$ & $2.7(2)$ \\
307 & $3.8(4)$ & $0.7(2)$ & $2.9(3)$ \\
317 & $7.0(6)$ & $1.0(3)$ & $3.1(3)$ \\
327 & $7.2(7)$ & $1.3(3)$ & $5.4(4)$ \\
\hline
\end{tabular}

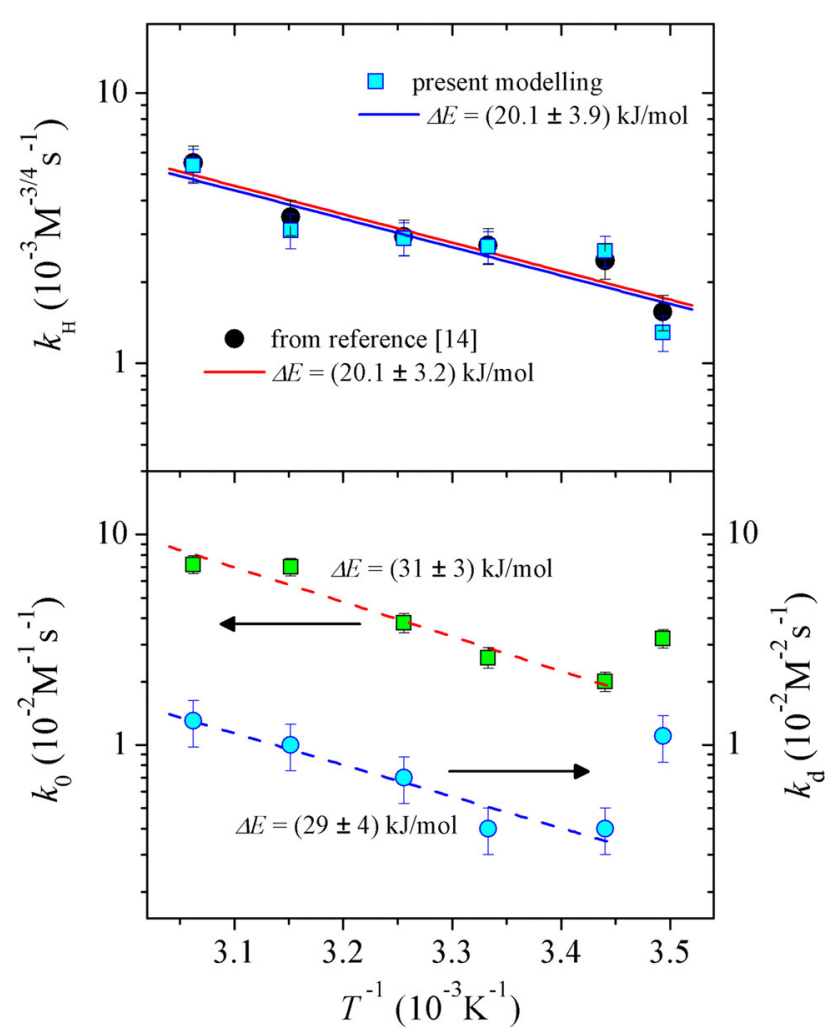

Fig. 2 (Above) Hydrolysis rate constants as obtained by fitting the present kinetic modeling (squares) in comparison with those (circles) as obtained earlier by a non-modeling method applied to the final homogeneous step of the reaction. (Below) Ultrasound and methanol producing dissolution rate constants $\left(k_{0}\right.$ and $\left.k_{\mathrm{d}}\right)$ as obtained by fitting the present kinetic modeling

dissolution process could be estimated from analyzing the Arrhenius-type plot of $k_{0}$ and $k_{\mathrm{d}}$ (at the high temperature side) in Fig. 2, for instance. We have found $\Delta E=(31 \pm 3) \mathrm{kJ} / \mathrm{mol}$ from $k_{0}$ and $\Delta E=(29 \pm 4) \mathrm{kJ} /-$ mol from $k_{\mathrm{d}}$ (Fig. 2), which are very similar values.

Besides the theoretical time function $\mathrm{d} C / \mathrm{d} t$ fitted and by extension $C$, the present modeling provides the theoretical virtually dissolved water $B_{\mathrm{S}}$ (and naturally $B=B_{0}-3$ $C-B_{\mathrm{s}}$ ) as a time function for each set of fitted rate constants $\left(k_{0}, k_{\mathrm{d}}\right.$ and $\left.k_{\mathrm{H}}\right)$. Assuming that the total water reacted at a time $t(3 C)$ is composed by a fraction $f$ come from the virtually dissolved water by ultrasound [by Eq. (2a)] and a fraction $(1-f)$ come from the dissolved water by methanol [by Eq. (2b)], the partial contributions to $B_{\mathrm{s}}$ due to the processes (2a), $B_{\text {sk0 }}$, and (2b), $B_{\text {skd }}$ could be evaluated separately by using Eqs. (3a) and (3b) as

$B_{\mathrm{sk} 0}=\int_{0}^{t} k_{0} A B \mathrm{~d} t-f 3 C$

and

$B_{\text {skd }}=\int_{0}^{t} k_{\mathrm{d}} D_{\mathrm{s}} A B \mathrm{~d} t-(1-f) 3 C$

where

$$
f=\int_{0}^{t} k_{0} A B \mathrm{~d} t /\left(\int_{0}^{t} k_{0} A B \mathrm{~d} t+\int_{0}^{t} k_{\mathrm{d}} D_{\mathrm{s}} A B \mathrm{~d} t\right) .
$$

Figure 3 shows the total virtually dissolved water $B_{\mathrm{s}}$ in comparison with the separated contributions to it due to the ultrasound-inducing dissolution $\left(B_{\mathrm{sk} 0}\right)$ and the methanol producing dissolution $\left(B_{\text {skd }}\right)$ as a time function. Figure 3 also shows the hydrolyzed quantity $C$ and the non-dissolved water $B$ (free water) as a time function.

The process of ultrasound-inducing dissolution was found to be much more effective to accelerate hydrolysis of GPTMS during the heterogeneous step of reaction (while $B$ was significantly larger than 0 ) in comparison with that of methanol producing dissolution, since $B_{\mathrm{sk} 0}$ was found too much larger than $B_{\text {skd }}$, even for the homogeneous step of the reaction starting at the immediacy of $B \cong 0$ (Fig. 3). So, ultrasound should be playing a more fundamental role on the virtual dissolution of water in GPTMS, even in presence of methanol, by fast mixing the reactant mixture. The more efficiency of ultrasound-inducing dissolution with respect to the alcohol-producing dissolution seems to be a general characteristic of the ultrasound-assisted hydrolysis of alkoxides formed by methoxy groups, as observed in the present GPTMS study and also in a previous work studying the ultrasound-assisted TMOS hydrolysis [18]. The specific process of alcohol-producing dissolution $\left(k_{\mathrm{d}}\right)$ seems to have its importance enhanced in the case of the ultrasound-assisted hydrolysis of TEOS [17], for instance, formed by ethoxy groups, likely because 


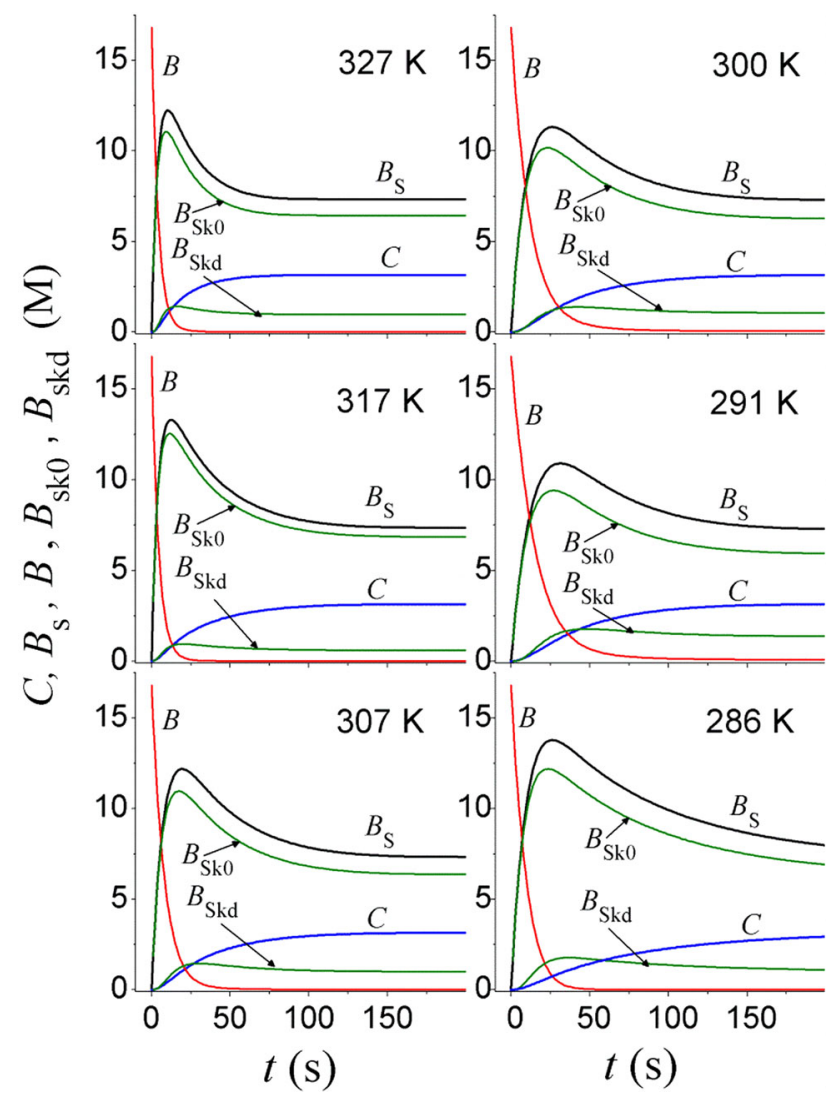

Fig. 3 Molar concentration as a time function in the GPTMS phase of the total virtually dissolved water $B_{\mathrm{s}}$, in comparison with the separated contributions to $B_{\mathrm{s}}$ due to the ultrasound-inducing dissolution $B_{\mathrm{sk} 0}$ and the methanol producing dissolution $B_{\mathrm{skd}}$. The molar concentrations of the hydrolyzed quantity $C$ and of the non-dissolved water $B$ (free water) are also shown as a time function

the hydrolysis rates of TEOS are typically much smaller than those of TMOS [20, 21]. Indeed, the hydrolysis of TMOS was found to be too rapid to be followed by a typical NMR study [20]. On the contrary, a heterogeneous mixture of TEOS and $\mathrm{HCl}$-water solution, for instance, is able to resist for a long time (about $6 \mathrm{~min}$ ) under ultrasound stimulation without to produce significant hydrolysis (a so-called induction period) [17]. In this case, ethanol yielded in the firstly hydrolysis [17], or even an initial addition of ethanol [22], determines the extension of the induction period, so emphasizing the enhancing of the relative importance of the alcohol-producing dissolution in the overall ultrasound-assisted hydrolysis of TEOS $[17,22]$.

A clear crossover from the heterogeneous to the homogeneous step of the reaction could not be obtained precisely with the present simplified modeling, since the condition $B \cong 0$ is attained only asymptotically with the present modeling, although $B$ was definitively found to drop sharply with the starting of the reaction, as shown in Fig. 3. The lack of such a clear crossover is expected since the present simplified model does not contemplate a discontinuous immiscibility gap line crossing.

The overall heterogeneous/homogeneous reaction pathway was analyzed in a ternary diagram as that conceived in Fig. 4 , where the molar fractions of GPTMS, $X_{\mathrm{GPTMS}}=A$ / $\left(A+B_{\mathrm{s}}+4 C\right)$, Bs $\left(\mathrm{H}_{2} \mathrm{O}\right.$ dissolved in GPTMS $)$, $X_{\mathrm{H}_{2} \mathrm{O}}=B_{\mathrm{s}} /\left(A+B_{\mathrm{s}}+4 C\right)$, and hydrolysis product $(\mathrm{C}+3 \mathrm{Ds}), \quad X_{\text {product }}=4 C /\left(A+B_{\mathrm{s}}+4 C\right), \quad$ have been plotted. The hydrolysis product $\left[\mathrm{R}-\mathrm{Si}(\mathrm{OH})_{3}+3 \mathrm{CH}_{3} \mathrm{OH}\right]$ has been assumed as a unique stable specie (product), with concentration $4 C$ in GPTMS phase, in plotting the ternary diagram of Fig. 4.

The typical overall heterogeneous/homogenous hydrolysis pathway plotted in Fig. 4 starts at the initial point $(1,0$, 0 ), in terms of the molar fraction notation $\left(X_{\mathrm{GPTMS}}, X_{\mathrm{H}_{2} \mathrm{O}}\right.$, $X_{\text {product }}$ ), where no water (Bs) is dissolved in GPTMS and no reacted quantity $(C)$ has been yielded, and ends at $(0$, $0.368,0.632$ ), where the hydrolysis has finished and 0.368 molar fraction of water in excess remained. Figure 4 shows that all the hydrolysis pathways associated with different temperatures converge to a unique pathway at an advanced stage of the reaction, which overlaps completely the trajectory we would observe if the reaction would be homogeneous in all its extension, the (red) straight line linking the point $(0.158,0.842,0)$ to $(0,0.368,0.632)$ in Fig. 4. The heterogeneous pathway portions in Fig. 4 were found, in general, shifting in direction to increase the dissolved water in the GPTMS phase with increasing temperature. The exception to this role was found for the sample hydrolyzed at the lowest temperature studied in the present work, as a result, as suggested earlier, of the better

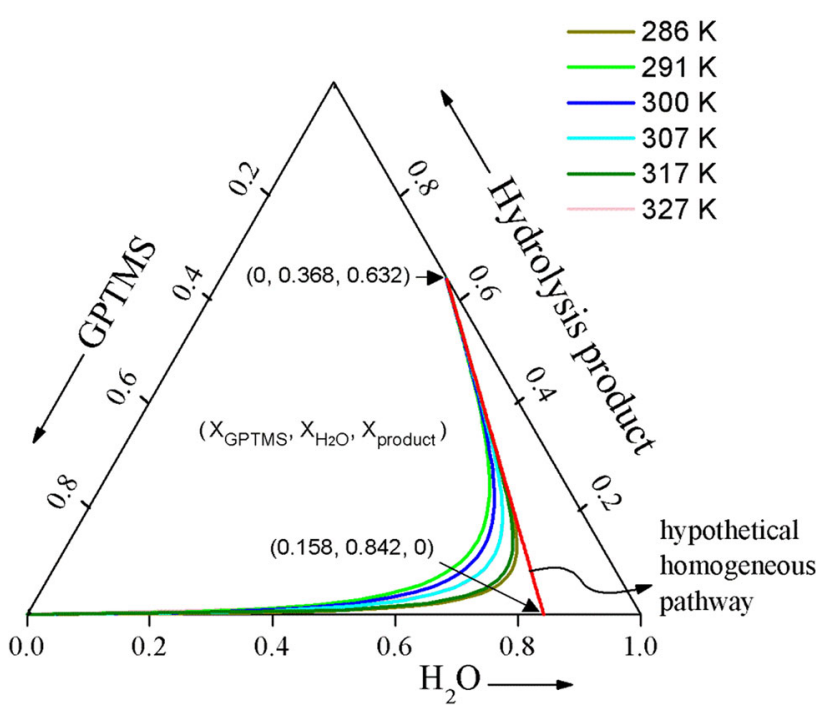

Fig. 4 Ternary diagram showing the overall heterogeneous/homogeneous hydrolysis reaction pathway in terms of the molar fractions of GPTMS, $\mathrm{H}_{2} \mathrm{O}$ dissolved in GPTMS, and the hydrolysis product $[\mathrm{R}-$ $\mathrm{Si}(\mathrm{OH})_{3}+3 \mathrm{CH}_{3} \mathrm{OH}$, being $\mathrm{R}$ the 3-glycidoxypropyl radical] (Color figure online) 
coupling between ultrasound and reactant medium at lower temperatures.

Finally, we wish to refer to the minor additional contribution to the experimental hydrolysis rate $\mathrm{d} C / \mathrm{d} t$, which is apparent in the plots of Fig. 1 at advanced stages of the homogeneous step of the reaction, mainly at lower temperatures. Such a minor experimental additional contribution is evidenced with respect to the fitted curves shown Fig. 1, mainly at lower temperatures. Based on the literature [20, 23, 24], we have argued [14, 17, 18, 22] that, because of the relatively rapid hydrolysis compared to the polycondensation reactions of such alkoxides under acid conditions and excess of water, the contribution of polycondensation reactions to the hydrolysis signal measured in the present calorimetric method could be neglected. In addition, an effect likes that minor additional contribution to the hydrolysis rates found in Fig. 1 for GPTMS was not found in the hydrolysis of TMOS in similar conditions [18]. The most evident difference between both alkoxides is the 3-glycidoxypropyl group in GPTMS substituting one of the methoxy groups of TMOS. So, we concluded that such an effect like that minor additional contribution to the experimental hydrolysis rates in the final homogeneous step of the reaction shown in Fig. 1 should be caused by some epoxy ring opening, even though it has been pointed that epoxy ring opening does not occur under acidified water $[25,26]$, not even under ultrasound stimulation [27, 28], except when catalyzed by amine (basic) groups $[25,29]$. Methanolysis of the epoxy groups is very plausible to be considered [30]; however, the epoxy ring opening has not been observed in methanol solution of a photocatalyst for epoxyketone reaction, even under ultrasound stimulation, though ultrasound was found to increase the rate of photoreaction in presence of UV radiation [28]. If the epoxy ring opening is occurring at some extension in the present GPTMS sono-hydrolysis, it could be due to some combined effect between the specific activity of the catalyst (a not so strong acid) and the ultrasound action. Oxalic acid (as a dibasic carboxylic acid) was observed to react with several epoxy resins causing effective epoxy ring opening in presence of basic catalysts $[15,16]$, although the epoxy ring opening was not so effective in the uncatalyzed reaction [16]. The incorporation of carboxy end groups in opened epoxy groups leads to additional cross-linking possibilities in structuring the resulting epoxy-oligomeric mixture [15]. So, the possibility of some epoxy ring opening in the present GPTMS system, in conjunction with temperature and continued ultrasound action, could be responsible for finding very different elastic properties in aged wet sonogels prepared from this set of the present GPTMS-derived sols hydrolyzed at different temperatures, as well correlated by the structural characteristics determined by small-angle X-ray scattering (SAXS) [14]. The structure of such a set of very aged wet sonogels was found to be composed by liquid-filled pores and an organic/ inorganic hybrid solid-phase, separated by a surface-fractal interface [14]. The hybrid solid-phase was constituted by polymeric linear chains, density fluctuation heterogeneities and several intermolecular-interfering cage-like structures, in proportions which were a temperature function of the earlier hydrolysis, so yielding aged sonogels with very different elastic properties [14], in such a way that aged sonogels hydrolyzed at lower temperature exhibited more elasticity, while those hydrolyzed at higher temperature were more rigid or more "crystallized" ones, according to the observation of a gradual increase and narrowing of a poor X-ray diffraction peak at about $1.5 \mathrm{~nm}$ as the hydrolysis temperature is increased [14]. The hypothesis of some epoxy ring opening in the present system allows us to explain more consistently the possibility to obtain final wet gels with different elastic properties by handling the proportions of polymeric linear chains, density fluctuation heterogeneities and intermolecular-interfering cage-like structures in the aged wet gels, by controlling the extension and characteristics of the epoxy ring opening reaction in the early hydrolysis. Indeed, the rate of epoxy ring opening reaction by oxalic acid in epoxy resins was found increasing with temperature [15], what could explain well the obtaining of more "crystallized" sonogels as higher the GPTMS hydrolysis temperature. However, it should be pointed that the epoxy ring opening possibility causes only a minor effect on the calorimetric experimental hydrolysis rates measurements, as shown in Fig. 1, so the experimental method remains valid and the applicability of the present kinetic modeling is not significantly compromised.

\section{Conclusions}

The experimental hydrolysis rates measured at several temperatures of the ultrasound-assisted and oxalic acidcatalyzed hydrolysis of GPTMS were satisfactorily fitted by a simplified dissolution and reaction modeling. The present kinetic modeling describes the overall heterogeneous/homogeneous pathway of the reaction in terms of dissolution of water in GPTMS, which increases the hydrolysis rate, followed by reaction in the GPTMS phase, which diminishes the hydrolysis rate as the reactants consumption.

Two parallel rate equations associated with the dissolution process of water in GPTMS have been considered: (1) One of them controlled by a second-order (on GPTMS and undissolved water) rate constant $k_{0}$ associated with water dissolution induced by ultrasound action; and (2) another controlled by a third-order (on GPTMS, undissolved water, and methanol) rate constant $k_{\mathrm{d}}$ associated with methanol producing dissolution. The ultrasound-inducing water dissolution was found to be the dominant 
mechanism controlling the dissolution process for the overall hydrolysis of GPTMS, when compared to that of the methanol producing dissolution.

The hydrolysis rate constants $k_{\mathrm{H}}$ (of $3 / 4$ order on GPTMS and first order on water) fitted by the present modeling to the overall heterogeneous/homogeneous pathway were found in good agreement with those obtained earlier using a non-modeling approach applied exclusively to the homogeneous step of the reaction.

The fitting of the present modeling put in evidence an additional minor contribution to the experimentally measured hydrolysis rates at an advanced degree of the homogeneous step of the reaction, which was attributed to some epoxy ring opening. This minor effect does not affect substantially the experimental hydrolysis rates signal, so the experimental method associated remains valid and the applicability of the present kinetic modeling assured.

Acknowledgments Research partially supported by FAPESP and CNPq, Brazil.

\section{References}

1. Innocenzi P, Figus $\mathrm{C}$, Kidchob $\mathrm{T}$, Valentine $\mathrm{M}$, Alonso $\mathrm{B}$, Takahashi M (2009) Dalton Trans 2009:9146-9152

2. Robertson MA, Rudkin RA, Parsonage D, Atkunson A (2003) J Sol-Gel Sci Technol 26:291-295

3. Liu YL, Su YH, Lai JY (2004) Polymer 45:6831-6837

4. Peng F, Liu L, Sun H, Wang Y, Liu J, Jiang Z (2005) Chem Mater 17:6790-6796

5. Deng TS, Zhang QF, Zhang JY, Shen X, Zhu KT, Wu JL (2009) J Colloid Interface Sci 329:292-299

6. Kang KS, Kim JH (2008) J Phys Chem C 112:618-620

7. Maly M, Posocco P, Fermeglia M, Pricl S (2008) Mol Simul 34:10-15

8. Boury B, Corriu R (2003) Chem Rec 3:120-132
9. Matějka L, Dukh O, Hlavatá D, Meissner B, Brus J (2001) Macromolecules 34:6904-6914

10. Menaa B, Takahashi M, Innocenzi P, Yoko T (2007) Chem Mater 19:1946-1953

11. Carboni D, Pinna A, Amenitsch H, Casula MF, Loche D, Malfattia L, Innocenzi P (2015) Phys Chem Chem Phys 17:10679-10686

12. de la Rosa-Fox N, Esquivias L, Craievich AF, Zarzycki J (1990) J Non-Cryst Solids 121:211-215

13. Ramirez-Del-Solar M, de la Rosa-Fox N, Esquivias L, Zarzycki J (1990) J Non-Cryst Solids 121:40-44

14. Paccola CET, Awano CM, de Vicente FS, Donatti DA, Yoshida M, Vollet DR (2015) J Phys Chem C 119:19162-19170

15. Bashta B, Astakhova O, Shyshchak O, Bratychak M (2014) Chem Chem Technol 8:309-316

16. Blank WJ, He ZA, Picci M (2002) J Coat Technol 74:33-41

17. Donatti DA, Ibañez-Ruiz A, Vollet DR (2002) Ultrason Sonochem 9:133-138

18. Donatti DA, Vollet DR, Ruiz AI (2000) J Sol-Gel Sci Technol 18:5-9

19. Goldberg RN, Kishore N, Lennen RM (2002) J Phys Chem Ref Data 31:231-370

20. Assink RA, Kay BD (1988) J Non-Cryst Solids 99:359-370

21. Pouxviel JC, Boilet JP, Beloeil JC, Lallemand JY (1987) J NonCryst Solids 89:345-360

22. Vollet DR, Donatti DA, Campanha JR (1996) J Sol-Gel Sci Technol 6:57-63

23. Artaki I, Sinha S, Irwin AD, Jonas J (1985) J Non-Cryst Solids 72:391-402

24. Yamane M, Inoue S, Yasumori A (1984) J Non-Cryst Solids 63:13-21

25. Davis SR, Brough AR, Atkinson A (2003) J Non-Cryst Solids 315:197-205

26. Innocenzi P, Brusatin G, Guglielmi M, Bertani R (1999) Chem Mater 11:1672-1679

27. Kamal A, Adil SF, Arifuddin MA (2005) Ultrason Sonochem $12: 429-431$

28. Memarian HR, Saffar-Teluri A (2007) Beilstein J Org Chem 3:2. doi:10.1186/1860-5397-3-2

29. Gizdavic-Nikolaidis MR, Edmonds NR, Bolt CJ, Easteal AJ (2008) Curr Appl Phys 8:300-303

30. Jeyakumar K, Chand DK (2008) Synthesis 5:807-819 